\title{
Amrubicin monotherapy may be an effective second-line treatment for patients with large-cell neuroendocrine carcinoma or high-grade non-small-cell neuroendocrine carcinoma
}

\author{
NORIMITSU KASAHARA ${ }^{1,2}$, KAZUSHIGE WAKUDA ${ }^{1}$, SHOTA OMORI $^{1}$, KAZUHISA NAKASHIMA $^{1}$, \\ AKIRA ONO $^{1}$, TETSUHIKO TAIRA ${ }^{1,3}$, HIROTSUGU KENMOTSU ${ }^{1}$, TATEAKI NAITO ${ }^{1}$, \\ HARUYASU MURAKAMI ${ }^{1}$, KEITA MORI $^{4}$, REIKO WATANABE ${ }^{5}$, MASAHIRO ENDO $^{6}$, \\ TAKASHI NAKAJIMA $^{5}$, MASANOBU YAMADA ${ }^{2}$ and TOSHIAKI TAKAHASHI ${ }^{1}$ \\ ${ }^{1}$ Division of Thoracic Oncology, Shizuoka Cancer Center, Nagaizumi, Shizuoka 411-8777; ${ }^{2}$ Department of Medicine \\ and Molecular Science, Gunma University Graduate School of Medicine, Maebashi, Gunma 371-8511; \\ ${ }^{3}$ Division of Respiratory Medicine, Minami Kyushu National Hospital, Aira, Kagoshima 899-5293; \\ ${ }^{4}$ Clinical Trial Coordination Office; ${ }^{5}$ Division of Diagnostic Pathology and ${ }^{6}$ Division of \\ Diagnostic Radiology, Shizuoka Cancer Center, Nagaizumi, Shizuoka 411-8777, Japan
}

Received November 14, 2016; Accepted January 30, 2017

DOI: $10.3892 / \mathrm{mco} .2017 .1198$

\begin{abstract}
There is no standard chemotherapy for pulmonary large-cell neuroendocrine carcinoma (LCNEC) and this type of cancer is difficult to diagnose using biopsy specimens. At the Shizuoka Cancer Center, when small biopsy specimens are used, they are diagnosed as high-grade non-small-cell neuroendocrine carcinoma (HNSCNEC) and the patients are treated according to the small-cell lung cancer (SCLC) guidelines. Amrubicin is an effective second-line treatment for patients with SCLC, although it remains unclear whether amrubicin monotherapy is effective for patients with LCNEC or HNSCNEC. Between September, 2004 and December, 2013, 18 patients with advanced LCNEC or HNSCNEC received amrubicin monotherapy in the second-line setting. The efficacy and toxicity of this treatment were retrospectively assessed. A total of 6 patients had LCNEC and 12 patients had HNSCNEC. The patients included 13 men, and the median age was 66 years (range, 57-82 years). A total of 16 patients had an Eastern Cooperative Oncology Group performance status of 0 or 1 . All the patients had received platinum-based chemotherapy as first-line treatment, and the median number of amrubicin cycles per patient was 4 (range, 1-9). The overall response rate was $11.1 \%$. The median progression-free and
\end{abstract}

Correspondence to: Dr Kazushige Wakuda, Division of Thoracic Oncology, Shizuoka Cancer Center, 1007 Shimonagakubo, Nagaizumi, Suntou-gun, Shizuoka 411-8777, Japan

E-mail:k.wakuda@scchr.jp

Key words: large-cell neuroendocrine carcinoma, high-grade non-small-cell neuroendocrine carcinoma, amrubicin, chemotherapy, second-line overall survival were 4.0 and 9.1 months, respectively. Grade 3 or 4 neutropenia was observed in $44 \%$ of the patients, and grade 3 febrile neutropenia occurred in $17 \%$ of the patients. One patient developed pneumonia and succumbed to the disease. Non-hematological toxicities were generally mild and manageable. Therefore, the efficacy of amrubicin in the second-line setting for patients with LCNEC or HNSCNEC is limited. Development of new drugs and/or treatment strategies is warranted.

\section{Introduction}

In 1980, pulmonary neuroendocrine tumors were categorized as typical carcinoid, atypical carcinoid, or small-cell lung carcinoma (1). However, in 1991, Travis et al introduced the idea of pulmonary large-cell neuroendocrine carcinoma (LCNEC); in 1999, the World Health Organization defined it as a variant of large-cell carcinoma, and this definition was retained in their 2004 guidelines (2-4). Neuroendocrine carcinomas of the lung are currently categorized as typical carcinoid, atypical carcinoid, LCNEC, or small-cell lung carcinoma. The majority of LCNEC cases are diagnosed via surgical specimens, since it is difficult to diagnose LCNEC using biopsy or cytology specimens, as biopsy specimens are too small to provide sufficient morphological information and to count the number of mitoses. Patients are diagnosed as possible LCNEC when it is difficult to establish a diagnosis of LCNEC, but there are no established criteria (5). Watanabe et al recently reported new diagnostic criteria for high-grade non-small-cell neuroendocrine carcinoma (HNSCNEC) as a surrogate for LCNEC in cases with a biopsy specimen (6). The HNSCNEC criteria include a Ki-67/MIB1 labeling index rather than morphological characteristics, as immunohistochemical staining may be performed on small samples that do no exhibit distinct morphological characteristics. These HNSCNEC criteria are 
used at the Shizuoka Cancer Center for cases with available biopsy specimens.

There is currently no standard chemotherapy for LCNEC. Rossi et al have reported that small-cell lung cancer (SCLC) regimens yield a median survival time (MST) of 51 months, and that non-SCLC (NSCLC) regimens yield an MST of 21 months, although these groups only included 12 and 15 patients, respectively (7). Based on these findings, LCNEC is treated using the SCLC strategy, and HNSCNEC is treated in the same manner. Niho et al have reported the efficacy of first-line cisplatin and irinotecan treatment for patients with LCNEC, although a central pathological review of their specimens revealed that they included SCLC cases (24\%), and that the response rate (RR) and the MST for LCNEC appeared to be inferior to those for SCLC (RR: 46.7 vs. 80\%, respectively, $\mathrm{P}=0.0823$; and MST: 12.6 vs. 17.3 months, respectively, $\mathrm{P}=0.047$ ) (8). Shimada et al have also demonstrated that the efficacy of second-line chemotherapy for possible LCNEC was inferior to that for SCLC (RR: 17 vs. 45\%, respectively) (9). Therefore, an effective second-line treatment agent is needed for patients with LCNEC. Amrubicin (AMR) is a fully synthetic 9-aminoanthracycline (a potent topoisomerase II inhibitor), and was reported to be effective in patients with SCLC and NSCLC. In a phase III study that compared AMR to topotecan for patients with SCLC in the second-line setting, AMR achieved an RR of $31.1 \%$, a median progression-free survival (PFS) of 4.1 months and an MST of 7.5 months (10). Furthermore, in a phase III study that compared AMR to docetaxel for patients with NSCLC in the second- or third-line setting, AMR exhibited an efficacy comparable to that of docetaxel (RR: 14.8 vs. $18.8 \%$, respectively, $\mathrm{P}=0.544$; PFS: 3.6 vs. 3.0 months, respectively, $\mathrm{P}=0.831$; and MST: 14.6 vs. 13.5 months, respectively, $\mathrm{P}=0.933$ ) (11). Moreover, Yoshida et al have reported the activity of AMR in patients with pretreated LCNEC (12), although $28 \%$ of the patients in that study had received $\geq 2$ chemotherapies prior to AMR treatment and the overall survival (OS) was 5.1 months. AMR may be effective in patients with LCNEC, although the availability of reports in the second-line setting is limited. Therefore, the aim of the present study was to retrospectively evaluate the efficacy of AMR in the second-line setting for patients with LCNEC or HNSCNEC.

\section{Materials and methods}

Criteria for diagnosing HNSCNEC. The criteria for diagnosing HNSCNEC comprise three major and three minor points (6). The major points are i) poorly differentiated NSCLC without acinar or squamous differentiation, with tumor cells containing a nucleus that is larger than the size of three small resting lymphocytes, a low nuclear:cytoplasmic ratio, or abundant cytoplasm, and tumor cell nucleus exhibiting a fine chromatin pattern and/or prominent nucleoli; ii) a Ki-67/MIB1 labeling index of $>40 \%$; and iii) positive immunostaining for $\geq 1$ neuroendocrine marker (neural cell adhesion molecule, chromogranin A and/or synaptophysin). The minor points are i) neuroendocrine morphology (organoid nesting, peripheral palisading, rosettes and trabecular architecture), ii) frequent massive necrosis and iii) intercellular space (cleft) or discohesiveness. The major points are essential for diagnosing
HNSCNEC, whereas the minor points indicate a higher likelihood of HNSCNEC. A Ki-67/MIB1 labeling index of $>40 \%$ confirms a high-grade tumor, and positive results for neuroendocrine markers confirm its neuroendocrine nature.

Patient selection. Patients were retrospectively selected according to the following criteria: i) A histological diagnosis of pulmonary LCNEC or HNSCNEC, ii) one prior chemotherapy treatment and ii) AMR monotherapy in the second-line setting.

Data collection. Patient characteristics were retrospectively collected from our institutional medical records. These characteristics included age, gender, smoking history, Eastern Cooperative Oncology Group performance status (PS), clinical stage, history of prior therapy, starting dose of AMR, number of cycles, tumor response, toxicities, date of recurrence and date of the last follow-up. The study protocol was approved by the Institutional Review Board of Shizuoka Cancer Center. Informed patient consent was waived due to the retrospective design of the study.

Evaluations. Clinical staging was performed using the 7 th version of the Union for International Cancer Control staging criteria (http://www.uicc.org/sites/main/files/private/TNM_ Classification_of_Malignant_Tumours_Website_15\%20MAy 2011.pdf). The best overall responses were classified according to version 1.1 of the Response Evaluation Criteria for Solid Tumor guidelines (https:/www.eortc.be/Recist/ documents/RECISTGuidelines.pdf). Toxicities were assessed using the 4th version of the Common Terminology Criteria for Adverse Events (https://evs.nci.nih.gov/ftp1/CTCAE/ CTCAE_4.03_2010-06-14_QuickReference_5x7.pdf).

Statistical analysis. OS was calculated from the date of AMR chemotherapy initiation to the date of death or the last follow-up. PFS was estimated from the date of AMR chemotherapy initiation to the date of recurrence or death. The Kaplan-Meier method was used to evaluate PFS and OS. All the statistical analyses were performed using JMP software, version 11.2 for Mac (SAS Institute, Cary, NC, USA).

\section{Results}

Patient characteristics. Between September, 2004 and December, 2013, 18 patients with advanced LCNEC or HNSCNEC received AMR monotherapy in the second-line setting. A total of 6 patients had LCNEC and 12 had HNSCNEC. Of the 6 LCNEC cases, 3 were diagnosed via surgically resected pulmonary specimens and 3 were diagnosed via specimens from brain metastases.

The patient characteristics are listed in Table I. The 18 patients included 13 men, and 17 of the 18 patients had a smoking history; the median patient age was 66 years (range, 57-82 years). A total of 3 patients experienced postoperative recurrence, and 13 patients had stage IV disease. The PS values were 0 or 1 for 16 patients, and 2 for the remaining 2 patients. The first-line chemotherapy regimens were cisplatin and irinotecan $(n=7)$, carboplatin and etoposide $(n=5)$, cisplatin and etoposide with thoracic irradiation $(n=2)$, 
Table I. Patient characteristics at the start of amrubicin treatment.

\begin{tabular}{|c|c|}
\hline Characteristics & Patients (n) \\
\hline \multicolumn{2}{|l|}{ Gender } \\
\hline Male & 13 \\
\hline Female & 5 \\
\hline \multicolumn{2}{|l|}{ Age (years) } \\
\hline Median & 66 \\
\hline Range & $57-82$ \\
\hline \multicolumn{2}{|l|}{ Smoking history } \\
\hline Current or former smoker & 17 \\
\hline Never smoked & 1 \\
\hline \multicolumn{2}{|l|}{ Clinical stage } \\
\hline Postoperative recurrence & 3 \\
\hline III & 2 \\
\hline IV & 13 \\
\hline \multicolumn{2}{|l|}{ Performance status } \\
\hline $0-1$ & 16 \\
\hline 2 & 2 \\
\hline \multicolumn{2}{|l|}{ Tumor histology } \\
\hline LCNEC & 6 \\
\hline HNSCNEC & 12 \\
\hline \multicolumn{2}{|l|}{ First-line chemotherapy regimen } \\
\hline Cisplatin + irinotecan & 7 \\
\hline Carboplatin + etoposide & 5 \\
\hline Cisplatin + etoposide + thoracic irradiation & 2 \\
\hline Cisplatin + etoposide & 2 \\
\hline Carboplatin + paclitaxel & 2 \\
\hline \multicolumn{2}{|l|}{ Starting dose of AMR (mg/m²/day) } \\
\hline 40 & 13 \\
\hline 35 & 4 \\
\hline 30 & 1 \\
\hline \multicolumn{2}{|l|}{ Number of subsequent chemotherapies } \\
\hline 3rd line & 10 \\
\hline 4th line & 4 \\
\hline 5th line & 2 \\
\hline
\end{tabular}

LCNEC, large-cell neuroendocrine carcinoma; HNSCNEC, high-grade non-small-cell neuroendocrine carcinoma; AMR, amrubicin.

cisplatin and etoposide $(\mathrm{n}=2)$ and carboplatin and paclitaxel $(n=2)$.

A total of 66 cycles of AMR chemotherapy were administered to the 18 patients. The 3-day starting dose of AMR was $40 \mathrm{mg} / \mathrm{m}^{2} /$ day for 13 patients, $35 \mathrm{mg} / \mathrm{m}^{2} /$ day for 4 patients, and $30 \mathrm{mg} / \mathrm{m}^{2} /$ day for 1 patient. There were two reasons for dose reductions, namely hepatic damage due to liver metastasis in 3 patients and old age in 2 patients. Any modifications during the subsequent cycles were determined according to the discretion of the attending physician. The median number of chemotherapy cycles per patient was 4 (range, 1-9). There were three reasons for early discontinuation of chemotherapy
Table II. Treatment-related toxicities with amrubicin in 18 previously treated patients with LCNEC or HNSCNEC.

\begin{tabular}{|c|c|c|c|c|c|}
\hline \multirow[b]{2}{*}{ Adverse events } & \multicolumn{5}{|c|}{ Grade (n) } \\
\hline & 1 & 2 & 3 & 4 & 5 \\
\hline \multicolumn{6}{|l|}{ Hematological } \\
\hline Leukopenia & 2 & 6 & 6 & 1 & 0 \\
\hline Neutropenia & 1 & 7 & 5 & 3 & 0 \\
\hline Anemia & 0 & 10 & 3 & 0 & 0 \\
\hline Thrombocytopenia & 0 & 1 & 0 & 0 & 0 \\
\hline \multicolumn{6}{|l|}{ Non-hematological } \\
\hline Febrile neutropenia & 0 & 0 & 3 & 0 & 0 \\
\hline Pneumonia & 0 & 0 & 0 & 0 & 1 \\
\hline Total bilirubin elevation & 0 & 1 & 1 & 0 & 0 \\
\hline AST elevation & 1 & 0 & 0 & 0 & 0 \\
\hline ALP elevation & 0 & 1 & 0 & 0 & 0 \\
\hline Hyponatremia & 0 & 0 & 1 & 0 & 0 \\
\hline Creatinine elevation & 4 & 0 & 0 & 0 & 0 \\
\hline Ileus & 0 & 0 & 1 & 0 & 0 \\
\hline Nausea & 4 & 4 & 1 & 0 & 0 \\
\hline Vomiting & 2 & 0 & 0 & 0 & 0 \\
\hline Anorexia & 8 & 3 & 1 & 0 & 0 \\
\hline Constipation & 4 & 1 & 0 & 0 & 0 \\
\hline Fatigue & 3 & 0 & 0 & 0 & 0 \\
\hline Alopecia & 0 & 1 & 0 & 0 & 0 \\
\hline Dysgeusia & 1 & 0 & 0 & 0 & 0 \\
\hline
\end{tabular}

LCNEC, large-cell neuroendocrine carcinoma; HNSCNEC, high-grade non-small-cell neuroendocrine carcinoma; AST, aspartate aminotransferase; ALP, alkaline phosphatase.

after only 1 cycle, namely disease progression in 4 patients, PS reduction in 1 patient and early death in 1 patient.

Efficacy. Of the 18 patients, 2 achieved a partial response (PR), 9 exhibited stable disease, 6 exhibited progressive disease, and 1 patient was not evaluated for tumor response due to a worsened general condition. The overall RR was $11.1 \%$ [95\% confidence interval (CI): 3.1-32.8\%], and the disease control rate (DCR) was $61.1 \%$ (95\% CI: 38.6-79.7\%]. The median PFS and MST were 4.0 months (95\% CI: 1.4-5.8 months) and 9.1 months (95\% CI: 2.2-15.7 months), respectively. Irinotecan monotherapy was the most frequently used third-line regimen, and only a few patients received fourth-line $(n=4)$ or fifth-line $(n=2)$ therapy.

Toxicity. The toxicities are listed in Table II. The most frequent toxicity was myelosuppression, which included grade 3 or 4 leukopenia (39\%), neutropenia (44\%) and anemia (17\%). Grade 3 febrile neutropenia was observed in $17 \%$ of the patients, and 1 patient died after developing pneumonia. Grade 2 and 3 total bilirubin elevation (each $n=1$ ), grade 1 aspartate aminotransferase elevation $(n=1)$ and grade 2 alkaline phosphatase elevation $(n=1)$ were also observed. 
Other non-hematological toxicities were generally mild and manageable.

\section{Discussion}

AMR has recently attracted attention as an active agent in the salvage setting, after a phase II study demonstrated that second-line AMR chemotherapy was effective for patients with SCLC. Furthermore, studies have reported an RR of 17-50\% and an MST of 5.3-10.3 months among patients with refractory relapsed SCLC, as well as an RR of 52-53\% and an MST of 9.9-11.6 months among patients with treatment-sensitive relapsed SCLC (13-16). A phase III trial also confirmed the efficacy of AMR as second-line treatment for patients with SCLC and second- or third-line treatment for patients with NSCLC $(10,11)$. These data indicate that AMR monotherapy is an effective agent in the salvage setting.

The present study revealed that second-line AMR monotherapy may be effective for LCNEC or HNSCNEC, with an RR of $11.1 \%$, a DCR of $61.1 \%$, a PFS of 4.0 months and an OS of 9.1 months. Yoshida et al have also reported that AMR monotherapy was effective for previously treated LCNEC, with an ORR of $27.7 \%$, a DCR of $61.1 \%$, a PFS or 3.1 months and an OS of 5.1 months (12). The RR of the present study was inferior to that of Yoshida et al, although the present study revealed a comparable DCR and an 2 -fold longer OS. These differences may be due to the relatively small sample size for each study and the differences in patient characteristics, as $28 \%$ of the patients had received $\geq 2$ chemotherapies prior to AMR treatment in the Yoshida study.

The main toxicity in the present study was myelosuppression, which included grade 3 or 4 neutropenia $(44 \%)$ and grade 3 febrile neutropenia (17\%). One death was also reported after the patient developed grade 5 pneumonia. That patient also experienced grade 4 neutropenia, which may have contributed to the fatal outcome. Bone marrow support may be required for the safe use of AMR. All other non-hematological toxicities were mild and manageable.

The present study included several limitations. First, the sample size was limited, although this is difficult to overcome, given the low incidence of LCNEC and HNSCNEC. Furthermore, our sample size was comparable to those of similar reports, and there are few reports regarding treatments for LCNEC, which we believe makes our findings valuable. Second, the timing of the patient evaluations may have differed for each physician, which may have affected the PFS and tumor response outcomes. However, these limitations are unavoidable, due to the retrospective nature of this study. Furthermore, OS is a reliable endpoint, and the MST of 9.1 months is favorable, compared with the findings of previous reports. Third, we cannot exclude the possibility of HNSCNEC combined with other subtypes (e.g., adenocarcinoma or SCLC), as our biopsy specimens only represent small parts of the tumor. However, in the advanced-stage setting, most diagnoses are based on bioptic specimens, and the treatment strategy does not change in the clinical setting, even if other organizational patterns are detected.

In conclusion, the efficacy of amrubicin in the second-line setting for patients with LCNEC or HNSCNEC is limited. Development of novel drugs and/or treatment strategies is warranted.

\section{Acknowledgements}

The present study was supported by a grant from the Japanese Agency for Medical Research and Development (no. 15ck0106131h0002). H.M. received honoraria from Nippon Kayaku Co., Ltd.; K.N. received speaking fees from Eli Lilly Japan, Ono Pharmaceutical. Co., Ltd, Taiho pharmaceutical Co., Ltd and Mochida Pharmaceutical Co., Ltd.; and M.E. received honoraria from AstraZeneca and Ono Pharmaceutical. Co., Ltd.

\section{References}

1. Arrigoni MG, Woolner LB and Bernatz PE: Atypical carcinoid tumors of the lung. J Thorac Cardiovasc Surg 64: 413-421, 1972.

2. Travis WD, Linnoila RI, Tsokos MG, Hitchcock CL, Cutler GB Jr, Nieman L, Chrousos G, Pass H and Doppman J: Neuroendocrine tumors of the lung with proposed criteria for large-cell neuroendocrine carcinoma. An ultrastructural, immunohistochemical, and flow cytometric study of 35 cases. Am J Surg Pathol 15: 529-553, 1991.

3. Travis W, Colby T, Corrin B, Shimosato Y and Brambilla E, eds: World health organization international histological classification of tumours: Histological typing of lung and pleural tumours, 3rd (eds). Berlin: Springer, 1999.

4. Travis W, Brambilla E, Muller-Hermelink H and Harris C, (eds): World health organization classification of tumours. Pathology and genetics: Tumours of the lung, pleura, thymus and heart. Lyon: IARC, 2004.

5. Travis WD, Brambilla E, Noguchi M, Nicholson AG, Geisinger KR, Yatabe Y, Beer DG, Powell CA, Riely GJ, Van Schil PE, et al: International association for the study of lung cancer/American thoracic society/European respiratory society international multidisciplinary classification of lung adenocarcinoma. J Thorac Oncol 6: 244-285, 2011.

6. Watanabe R, Ito I, Kenmotsu H, Endo M, Yamamoto N, Ohde Y, Kondo H, Nakajima T and Kameya T: Large cell neuroendocrine carcinoma of the lung: Is it possible to diagnose from biopsy specimens? Jpn J Clin Oncol 43: 294-304, 2013.

7. Rossi G, Cavazza A, Marchioni A, Longo L, Migaldi M, Sartori G, Bigiani N, Schirosi L, Casali C, Morandi U, et al: Role of chemotherapy and the receptor tyrosine kinases KIT, PDGFRalpha, PDGFRbeta, and Met in large-cell neuroendocrine carcinoma of the lung. J Clin Oncol 23: 8774-8785, 2005.

8. Niho S, Kenmotsu H, Sekine I, Ishii G, Ishikawa Y, Noguchi M, Oshita F, Watanabe S, Nakajima R, Tada $H$ and Nagai $K$ : Combination chemotherapy with irinotecan and cisplatin for large-cell neuroendocrine carcinoma of the lung: A multicenter phase II study. J Thorac Oncol 8: 980-984, 2013.

9. Shimada Y, Niho S, Ishii G, Hishida T, Yoshida J, Nishimura M, Yoh K, Goto K, Ohmatsu H, Ohe Y and Nagai K: Clinical features of unresectable high-grade lung neuroendocrine carcinoma diagnosed using biopsy specimens. Lung Cancer 75: 368-373, 2012.

10. von Pawel J, Jotte R, Spigel DR, O'Brien ME, Socinski MA, Mezger J, Steins M, Bosquée L, Bubis J, Nackaerts K, et al: Randomized phase III trial of amrubicin versus topotecan as second-line treatment for patients with small-cell lung cancer. J Clin Oncol 32: 4012-4019, 2014.

11. Katakami N, Yoshioka H, Okamoto H, Iwamoto Y, Seto T, Takahashi T, Sunaga N, Kudoh S, Chikamori K, Harada M, et al: Amrubicin (AMR) versus docetaxel (DTX) as second- or third-line treatment for non-small cell lung cancer (NSCLC): A randomized phase III trial. Ann Oncol 25 (Suppl 4): iv426-iv470, 2014.

12. Yoshida H, Sekine I, Tsuta K, Horinouchi H, Nokihara H, Yamamoto N, Kubota K and Tamura T: Amrubicin monotherapy for patients with previously treated advanced large-cell neuroendocrine carcinoma of the lung. Jpn J Clin Oncol 41: 897-901, 2011.

13. Onoda S, Masuda N, Seto T, Eguchi K, Takiguchi Y, Isobe H, Okamoto H, Ogura T, Yokoyama A, Seki N, et al: Phase II trial of amrubicin for treatment of refractory or relapsed small-cell lung cancer: Thoracic oncology research group study 0301 . J Clin Oncol 24: 5448-5453, 2006. 
14. Ettinger DS, Jotte R, Lorigan P, Gupta V, Garbo L, Alemany C, Conkling P, Spigel DR, Dudek AZ, Shah C, et al: Phase II study of amrubicin as second-line therapy in patients with platinum-refractory small-cell lung cancer. J Clin Oncol 28: 2598-2603, 2010.

15. Inoue A, Sugawara S, Yamazaki K, Maemondo M, Suzuki T, Gomi K, Takanashi S, Inoue C, Inage M, Yokouchi H, et al: Randomized phase II trial comparing amrubicin with topotecan in patients with previously treated small-cell lung cancer: North Japan lung cancer study group trial 0402. J Clin Oncol 26: 5401-5406, 2008.
16. Murakami H, Yamamoto N, Shibata T, Takeda K, Ichinose Y, Ohe Y, Yamamoto N, Takeda Y, Kudoh S, Atagi S, et al: A single-arm confirmatory study of amrubicin therapy in patients with refractory small-cell lung cancer: Japan clinical oncology group study (JCOG0901). Lung Cancer 84: 67-72, 2014. 